\title{
LOS MATRIMONIOS ILEGALES DEL ARTÍCULO 217 DEL CÓDIGO PENAL, MENCIÓN ESPECIAL AL DOLO Y AL ERROR DE TIPO
}

Francisco Martinez Rivas

Profesor Asociado del Departamento de Historia Jurídica y de Ciencias Penales y Criminológicas

E-mail: $\underline{\text { fmr3@um.es }}$

\begin{abstract}
RESUMEN: Los matrimonios ilegales, y más concreto el previsto en el artículo 217 del Código penal, sigue estando de actualidad sobre todo en los con denominados matrimonios de conveniencia. Especial consideración tiene el elemento subjetivo que concurre en el mencionado delito, amén de la la cuestión referida a la existencia posible de error de tipo penal.

Cuestiones relativas al bien jurídico protegido, la descripción de la conducta delictiva, el lugar de comisión del delito, así como un exhaustivo análisis jurisprudencial, vienen a vertebrar el presente trabajo que, lejos de considerar que se trata de un delito vetusto, se encuentra más en la vida diaria de lo que podemos considerar.
\end{abstract}

Palabras clave: Matrimonio ilegal; bien jurídico protegido; dolo; lugar de comisión.

ABSTRACT: Illegal marriages, and more specifically the one provided for in Article 217 of the Criminal Code, are still topical, especially in socalled marriages of convenience. Special consideration must be given to the subjective element involved in this offence, in addition to the question of the possible existence of criminal misconduct.

Questions relating to the protected legal right, the description of the criminal conduct, the place of commission of the crime, as well as an exhaustive jurisprudential analysis, form the backbone of this work which, far from considering it to be an outdated crime, is more common in everyday life than we might think.

Keywords: Unlawful marriage; protected legal interest; intent; place of commission. 


\section{PLANTEAMIENTO GENERAL}

El delito de contraer un segundo o ulterior matrimonio a sabiendas de la subsistencia de un matrimonio legalmente celebrado con anterioridad, al margen de que pudiera tener una íntima relación con el delito de usurpación de estado civil, previsto en el art. $401 \mathrm{CP}$, es claro que el art. $217 \mathrm{CP}$ supone la génesis de un estado civil aparente. Ese estado civil aparente nace "viciado" ab initio, y por lo tanto es nulo.

Indica el art. $217 \mathrm{CP}$ que "[e]l que contrajere segundo o ulterior matrimonio, a sabiendas de que subsiste legalmente el anterior, será castigado con la pena de prisión de seis meses a un año".

Sin especial deseo en hacer menciones históricas acerca de la penología del tipo, es lo cierto que la misma se ha visto convenientemente mitigada a lo largo de la historia de nuestro Derecho penal. Desde la redacción del Código Penal de 1850 que preveía en este tipo de delitos la penal capital ${ }^{1}$ hasta la prisión máxima de un año que prevé nuestro actual Código Penal de 1995, la importancia de este tipo ha ido cayendo en desuso, incluso, como se dirá, la suspensión de la condena impuesta por la meritada conducta penalmente relevante suele ser el final de la misma. Los motivos de remisión de la penal por la vía del art. $80 \mathrm{CP}$, por lo general es, además de por tratarse de una penal inferior a dos años, por la concurrencia en el sujeto activo de la ausencia de antecedentes a este respecto, por la escasa peligrosidad de la conducta, además de por las exiguas posibilidades de reincidencia del sujeto activo.

La problemática, o más bien, la errónea consideración tradicional (hoy ya superada) como sujeto pasivo del delito en la persona del cónyuge contrayente de ese segundo matrimonio (como he indicado nulo ab initio), subsistiendo el primero de ellos, ha sido resuelta. En ningún caso, salvo auténticos contrayentes de buena fe, puede considerarse sujeto pasivo al otro contrayente ${ }^{2}$ que, o bien contrae nupcias sabiendo de la existencia de ese matrimonio primigenio, bien sin interés alguno en la certeza de la soltería del sujeto activo, en tanto que se trate de un matrimonio de conveniencia. En este caso, el sujeto contrayente (sin vínculo válido anterior) podría ser considerado también sujeto activo en calidad de cómplice (art. 29 $\mathrm{CP}$ ) o, incluso, cooperador necesario (art. 28 b), en su caso. $\mathrm{O}$ incluso en ocasiones junto a la bigamia pueden aparecer otros delitos en los que uno de los cónyuges sea el sujeto pasivo, como por ejemplo el delito de abandono de familia ${ }^{3}$, o el de estafa ${ }^{4}$.

1 Vid. sobre este punto LABACA ZABALA, M.L., "El delito de bigamia", Revista Electrónica Derecho Penal Online (disponible en http://www.derechopenalonline.com), donde la autora hace un recorrido por la progresiva reducción de la pena impuesta a este delito en los sucesivos Códigos Penales vigentes en España, desde la citada pena capital (contenida en el CP de 1850) hasta la prisión máxima de un año que establece el CP de 1995.

2 TRAPELO BARREALES, M.A. "Matrimonios ilegales y derecho penal. Bigamia, matrimonio inválido, matrimonio de conveniencia, matrimonio forzado y matrimonio precoz. Los delitos de matrimonios ilegales: Arts. 217 y 218 CP". Tiranto Lo Blanch. Marzo 2016, pp. 4 y ss.

3 El incumplimiento de los deberes de asistencia inherentes a la patria potestad, tutela, guarda o acogimiento familiar. Para que sea sancionado penalmente, ha de ser grave y continuado (STS 730/2011, de 12 de julio). O bien dejar de prestar la asistencia legalmente establecida para el sustento de descendientes y cónyuges en este caso.

4 BOIX REIG/JAREÑO LEAL, en: VIVES ANTÓN (coord.), Comentarios CP I, 1996, 1047 s., analizando el concurso con la estafa y las falsedades; SUÁREZ GONZÁLEZ, en: RODRÍGUEZ MOURULLO (dir.)/JORGE BARREIRO (coord.), Comentarios CP, 1997, 646, analizando el concurso con los delitos de falsedades documentales; CARBONELL MATEU, en: H-Vidal Guitarte I, 1999, 174, analizando el concurso con la estafa y las falsedades documentales; CARBONELL MATEU/GONZÁLEZ CUSSAC, en: VIVES ANTÓN/ORTS BERENGUER/CARBONELL MATEU/GONZÁLEZ CUSSAC/MARTÍNEZ-BUJÁN PÉREZ, PE, 3ª 2010, 
Sin embargo, y como ha puesto de manifiesto de forma brillante M.A. TRAPELO BARREALES $^{5}$, de la consideración del delito de bigamia como delito especial propio pueden derivarse varias situaciones problemáticas.

La primera tiene que ver con la posible responsabilidad penal del contrayente no bígamo que sabe que contrae matrimonio con una persona sobre la que existe el impedimento no dirimente de vínculo.

La segunda, cuando el bígamo desconoce esta circunstancia, que va a contraer un matrimonio sin saber y/o conocer que subsiste otro matrimonio legalmente contraído por él mismo, pero de este hecho sí es conocedor el segundo contrayente. En este caso, se ha considerado que la conducta del bígamo no es punible por falta de tipicidad, ya que

381, analizando el concurso con la estafa; MORETÓN TOQUERO, Matrimonios ilegales, 2001, 17, planteando el concurso con abandono de familia (y con el de falsedades mencionando las dudas sobre el tratamiento concursal); MIR PUIG, en: EPB 2002, 192, mencionando las relaciones concursales con las falsedades, abandono de familia, estafa y abusos sexuales, y parece defender la tesis de que se aprecien las reglas del concurso de leyes, a través del principio de consunción a favor del delito de bigamia; TERRADILLOS BASOCO, en: COBO DEL ROSAL (dir.), Comentarios CP VII, 2003, 681; en: TERRADILLOS BASOCO (coord.), PE I, 2011, 302, analizando el concurso con la estafa y la falsedad documental, en este caso analizando la tesis jurisprudencial sobre la calificación del concurso como de leyes cuando la falsedad documental es posterior a la comisión del delito de bigamia; CUGAT MAURI, en: CÓRDOBA RODA/GARCÍA ARÁN (dirs.), Comentarios CP PE I, $2004,544,551$, analizando el concurso con la estafa y el abandono de familia, mientras que con las falsedades documentales se trata de un concurso de leyes (también cuando la falsedad es previa a la celebración del segundo matrimonio); MUÑOZ SÁNCHEZ, en: DÍEZ RIPOLLÉS/ROMEO CASABONA (coords.), Comentarios CP II, 2004, 1076 ss., analizando las relaciones concursales con el abandono de familia (de leyes, a favor de la bigamia por principio de consunción), la estafa (concurso ideal) y las falsedades (concurso medial cuando la falsedad es instrumental, lo que lleva a un resultado penológico revelador de la necesaria destipificación del delito de bigamia); SAAVEDRA RUIZ, en: CONDE-PUMPIDO TOURÓN (dir.)/LÓPEZ BARJA DE QUIROGA (coord.), CP III, 2007, 1650, analizando el concurso con la falsedad documental (a favor del concurso de leyes para evitar la excesiva punibilidad de aplicarse las reglas del concurso de delitos); CARRASCO ANDRINO, en: LLP 66 (2009), 14 s., analizando los concursos con las falsedades (de delitos si la falsedad es previa a la bigamia, de leyes si es posterior), abandono de familia y estafa; en: ÁLVAREZ GARCÍA (dir.)/MANJÓN-CABEZA OLMEDA/ VENTURA PÜSCHEL (coords.), PE I, 2a , 2011, 879; en: QUINTERO OLIVARES/CARBONELL MATEU/ MORALES PRATS/GARCÍA RIVAS/ÁLVAREZ GARCÍA (dirs.)/MANJÓN-CABEZA OLMEDA/VENTURA PÜSCHEL (coords.), PE, 2011, 231; MANZANARES SAMANIEGO, CP II, 2010, 363, planteando el concurso con la estafa, las falsedades documentales y los delitos contra la libertad sexual; GÓMEZ MARTÍN, en: CORCOY BIDASOLO/MIR PUIG (dirs.), Comentarios CP, 2011, 496, analizando el concurso con el delito de abandono de familia (concurso de leyes a favor del delito de bigamia por principio de consunción), en: CORCOY BIDASOLO (dir.)/VERA SÁNCHEZ (coord.), PE I, 2015, 449; GORDILLO ÁLVAREZ-VALDÉS, en: LAMARCA PÉREZ (coord.), $P E, 6$ a $, 2011,229$, planteando el concurso con la estafa y las falsedades documentales (cuando la falsedad es medio para cometer la bigamia); RAMÓN RIBAS, en: QUINTERO OLIVARES (dir.)/MORALES PRATS (coord.), $P E, 9^{\text {a }}, 2011,558$ s., 560, planteando el concurso con los delitos de estafa y abandono de familia (y en estos delitos el nuevo cónyuge sería sujeto pasivo del delito); BOIX REIG, en: BOIX REIG (dir.), PE II, 2012, 20, analizando el concurso con las falsedades documentales (valorando si el concurso se tiene que resolver con las reglas del concurso de leyes o de delitos); MENDOZA BUERGO, en: MOLINA FERNÁNDEZ (coord.), Memento práctico penal, 2015, 1023/nm. 10327, analizando el concurso con el abandono de familia y las falsedades (si son previas a la celebración del segundo matrimonio); MUÑOZ CONDE, $P E, 20^{\mathrm{a}}, 2015,272$, desde el delito de estafa (el nuevo cónyuge sería sujeto pasivo); PÉREZ CEPEDA, en: GÓMEZ RIVERO (dir.), $P E I, 2^{\text {a }}, 2015,353$ (desde el delito de estafa, y el nuevo cónyuge sería sujeto pasivo del delito); QUERALT JIMÉNEZ, $P E, 7^{\text {a }}, 2015,398$, analizando el concurso con la estafa, el abandono de familia y las falsedades; RODRÍGUEZ NÚÑEZ, en: LAMARCA PÉREZ (coord.), PE, 3ª , 2015, 264, planteando el concurso con la estafa y las falsedades documentales.

5 Vid. TRAPELO BARREALES, M.A. "Matrimonios ilegales y derecho penal. Bigamia, matrimonio inválido, matrimonio de conveniencia, matrimonio forzado y matrimonio precoz. Los delitos de matrimonios ilegales: Arts. 217 y 218 CP". Tiranto Lo Blanch. Marzo 2016, pp. 8 y ss. 
no actúa dolosamente (carece del dolo de primer grado), atipicidad que repercute en el otro contrayente, ya que en virtud de la accesoriedad (limitada) de la participación estaría participando en un hecho atípico.

Esta impunidad del segundo contrayente no puede determinarse en atención a que pudiera ser un autor mediato, ya que conoce la situación de bigamia, y lo más importante, le falta la condición o cualidad especial para ser autor del delito y, en consecuencia, también para poder realizar la conducta típica (pues no contrae segundo matrimonio) ${ }^{6}$.

La tercera, cuando los dos contrayentes del segundo o ulterior matrimonio han contraído nupcias cada uno de ellos, con otros cónyuges, un matrimonio con anterioridad, y ambos matrimonios subsisten legalmente. En este supuesto se plantea la cuestión de si se han cometido uno o dos delitos, y si los bígamos conocen esta circunstancia, en el segundo caso cada contrayente respondiendo como autor de su delito de bigamia y como partícipe en el delito de bigamia del otro contrayente.

Esta última es la calificación planteada por un sector de la doctrina, recurriendo a las reglas del concurso ideal entre dos delitos de bigamia en relación con cada uno de los contrayentes, uno cometido a título de autor, el otro cometido como partícipe ${ }^{7}$. Solución que parece es la que debe mantenerse atendiendo a la formulación del bien jurídico protegido. El hecho de que el matrimonio consista en la unión de dos personas no puede significar que, en este caso, se esté ante un caso de coautoría; cada contrayente ha de otorgar su consentimiento matrimonial para que el matrimonio se declare contraído, pero si los dos contrayentes están casados con anterioridad, cada uno de ellos contrae matrimonio subsistiendo legalmente uno anterior (uno por cada contrayente).

6 Llegan a la conclusión de que no cabe castigar al contrayente que conoce que se casa con un bígamo, siendo este desconocedor de tal circunstancia, a través de las reglas de la autoría mediata, entre otros, MIR PUIG, en: $A D P C P$ 1974, 468; CARBONELL MATEU, en: H-Vidal Guitarte I, 1999, 174; CARBONELL MATEU/GONZÁLEZ CUSSAC, en: VIVES ANTÓN/ORTS BERENGUER/CARBONELL MATEU/GONZÁLEZ CUSSAC/ MARTÍNEZ-BUJÁN PÉREZ, $P E$, 3a , 2010, 381; CARBONELL MATEU, en: GONZÁLEZ CUSSAC (coord.), $P E, 4^{\text {a }}, 2015,314$; TERRADILLOS BASOCO, en: COBO DEL ROSAL (dir.), Comentarios CP VII, 2003, 679; en: ARROYO ZAPATERO/BERDUGO GÓMEZ DE LA TORRE/FERRÉ OLIVÉ/GARCÍA RIVAS/SERRANO PIEDECASAS/TERRADILLOS BASOCO (dirs.)/NIETO MARTÍN/PÉREZ CEPEDA (coords.), Comentarios $C P$, 2007, 494; en: TERRADILLOS BASOCO (coord.), PE I, 2011, 301; CARRASCO ANDRINO, en: LLP 66 (2009), 9; en: ÁLVAREZ GARCÍA (dir.)/MANJÓN-CABEZA OLMEDA/VENTURA PÜSCHEL (coords.), $P E$ I, 2 a 2011, 873 s.; en: QUINTERO OLIVARES/CARBONELL MATEU/MORALES PRATS/GARCÍA RIVAS/ ÁLVAREZ GARCÍA (dirs.)/MANJÓN-CABEZA OLMEDA/VENTURA PÜSCHEL (coords.), PE, 2011, 230; QUERALT JIMÉNEZ, PE, 7 , 2015, 396.

7 Defienden esta tesis, entre otros, BOIX REIG/JAREÑO LEAL, en: VIVES ANTÓN (coord.), Comentarios CP I, 1996, 1046, 1047; SUÁREZ GONZÁLEZ, en: RODRÍGUEZ MOURULLO (dir.)/JORGE BARREIRO (coord.), Comentarios CP, 1997, 646; MUÑOZ SÁNCHEZ, en: DÍEZ RIPOLLÉS/ROMEO CASABONA (coords.), Comentarios CP II, 2004, 1074; CHOCLÁN MONTALVO, en: CALDERÓN CEREZO/CHOCLÁN MONTALVO,PE, 2005, 182; SAAVEDRA RUIZ, en: CONDE-PUMPIDO TOURÓN (dir.)/LÓPEZ BARJA DE QUIROGA (coord.), Comentarios CP III, 2007, 1649; MANZANARES SAMANIEGO, CP II, 2010, 363; GORDILLO ÁLVAREZ-VALDÉS, en: LAMARCA PÉREZ (coord.), PE, 6a , 2011, 229; BOIX REIG, en: BOIX REIG (dir), PE II, 2012, 20; RODRÍGUEZ NÚÑEZ, en: LAMARCA PÉREZ (coord.), PE, 3a, 2015, 264. MORETÓN TOQUERO, Matrimonios ilegales, 2001, 15; CARDONA TORRES, PE, 2010, 211; GONZÁLEZ RUS, en: MORILLAS CUEVA (coord.), PE, 2011, 377; SUÁREZ-MIRA RODRÍGUEZ, en: SUÁREZ-MIRA RODRÍGUEZ (coord.), $P E, 6^{\text {a }}, 2011,222$; QUERALT JIMÉNEZ, $P E, 7^{\text {a }}, 2015,398$. Para CARRASCO ANDRINO, en: LLP 66 (2009), 8 s.; en: ÁLVAREZ GARCÍA (dir.)/MANJÓN-CABEZA OLMEDA/VENTURA PÜSCHEL (coords.), PE I, 2a , 2011, 873 s.; en: QUINTERO OLIVARES/CARBONELL MATEU/MORALES PRATS/GARCÍA RIVAS/ÁLVAREZ GARCÍA (dirs.)/MANJÓN-CABEZA OLMEDA/VENTURA PÜSCHEL (coords.), PE, 2011, 230. 
Para concluir con la exposición sobre el sujeto activo, dada la naturaleza del matrimonio, y las características que ha de reunir el consentimiento matrimonial para que surta el efecto que le es propio, no cabe la posibilidad de plantear supuestos de autoría mediata. Pues aun cuando se plantee un supuesto de matrimonio por poderes (art. $55 \mathrm{CC}$ ) quien contrae el matrimonio es el representado, pues él es quien está manifestando el consentimiento matrimonial a través de su representante.

\subsection{El problema de la determinación del bien jurídico protegido}

Respecto al bien jurídico protegido, como se explicará, no existe una opinión unánime al respecto, ni siquiera pacífica, al igual que la concreción del sujeto pasivo. Por lado, un parte de la doctrina estima que se trata de un bien jurídico colectivo ${ }^{8}$, teniendo la condición de perjudicados, en su caso, el cónyuge anterior y el aparente nuevo cónyuge (siempre que haya contraído nupcias de buena fe) ${ }^{9}$.

Para otro sector doctrinal, el bien jurídico protegido es colectivo también, aunque vienen a precisar el mismo. Viene a ser considerado como tal, por ejemplo; la protección de la familia con relación a los derechos y deberes que derivan de la misma ${ }^{10}$. En otras opiniones, el bien jurídico protegido se vertebra en el estado civil y las relaciones fami-

8 Defienden que el sujeto pasivo es exclusivamente la sociedad, entre otros, BOIX REIG/JAREÑO LEAL, en: VIVES ANTÓN (coord.), Comentarios CP I, 1996, 1046; CARBONELL MATEU, en: H-Vidal Guitarte I, 1999, 174; CARBONELL MATEU/GONZÁLEZ CUSSAC, en: VIVES ANTÓN/ORTS BERENGUER/CARBONELL MATEU/GONZÁLEZ CUSSAC/MARTÍNEZ-BUJÁN PÉREZ,PE, 3a , 2010, 381，383; CARBONELL MATEU, en: GONZÁLEZ CUSSAC (coord.), $P E$, 4a $, 2015,315$; TERRADILLOS BASOCO, en: COBO DEL ROSAL (dir.), Comentarios CP VII, 2003, 679; en: TERRADILLOS BASOCO (coord.), PE I, 2011, 299, 301; CARRASCO ANDRINO, en: LLP 66 (2009), 7; en: ÁLVAREZ GARCÍA (dir.)/MANJÓN-CABEZA OLMEDA/VENTURA PÜSCHEL (coords.), PE I, 2a , 2011, 874; en: QUINTERO OLIVARES/CARBONELL MATEU/MORALES PRATS/GARCÍA RIVAS/ÁLVAREZ GARCÍA (dirs.)/MANJÓN-CABEZA OLMEDA/ VENTURA PÜSCHEL (coords.), PE, 2011, 230; GONZÁLEZ RUS, en: MORILLAS CUEVA (coord.), $P E$, 2011, 376, 377; RAMÓN RIBAS, en: QUINTERO OLIVARES (dir.)/MORALES PRATS (coord.),PE, 9a , 2011, 560, 562; MENDOZA BUERGO, en: MOLINA FERNÁNDEZ (coord.), Memento práctico penal, 2015, 1020/ nm. 10315 .

9 Así lo mantienen, entre otros, CUGAT MAURI, en: CÓRDOBA RODA/GARCÍA ARÁN (dirs.), Comentarios CP PE I, 2004, 547; CARRASCO ANDRINO, en: LLP 66 (2009), 7 s.; en: ÁLVAREZ GARCÍA (dir.)/MANJÓNCABEZA OLMEDA/VENTURA PÜSCHEL (coords.), PE I, 2 $2^{\mathrm{a}}, 2011,874$; en: QUINTERO OLIVARES/ CARBONELL MATEU/MORALES PRATS/GARCÍA RIVAS/ÁLVAREZ GARCÍA (dirs.)/MANJÓN-CABEZA OLMEDA/VENTURA PÜSCHEL (coords.), PE, 2011, 230; MARTÍNEZ GARCÍA, en: GÓMEZ TOMILLO (dir.), Comentarios $C P, 2^{\mathrm{a}}$, 2011, 852; RAMÓN RIBAS, en: QUINTERO OLIVARES (dir.)/MORALES PRATS (coord.), $P E, 9^{a}$, 2011, 560; MENDOZA BUERGO, en: MOLINA FERNÁNDEZ (coord.), Memento práctico penal, 2015, 1022/nm. 10320. Aun cuando se acepta que el bien jurídico protegido es el matrimonio monogámico, también se ha considerado como sujeto pasivo del delito, además de la colectividad, al otro contrayente de buena fe. Esta es la opinión defendida por QUERALT JIMÉNEZ, PE, 7ª , 2015, 396; RODRÍGUEZ NÚÑEZ, en: LAMARCA PÉREZ (coord.), $P E, 3^{\mathrm{a}}, 2015,262$. Desde la consideración de que el objeto de protección es el estado civil, proponen como sujeto pasivo del delito al otro contrayente GARCÍA VALDÉS/MESTRE DELGADO/FIGUEROA NAVARRO, $P E, 2^{\mathrm{a}}, 2015,101$. Se muestra vacilante SERRANO TÁRRAGA, en: SERRANO GÓMEZ/SERRANO MAÍLLO/ SERRANO TÁRRAGA/VÁZQUEZ GONZÁLEZ, PE, 2012, 216, si bien finalmente parece aceptar que el sujeto pasivo es la comunidad, pues a ella interesa mantener el modelo de matrimonio monógamo. Y a continuación añade que en algunos casos también podría ser sujeto pasivo el contrayente del segundo matrimonio si actuó de buena fe. No queda suficientemente clara la calificación del sujeto pasivo del delito de bigamia (entonces tipificado en el art. 471 CP) en la STS 31-1-1986, pues en la valoración de este elemento se afirma que, desde luego, la persona ligada por el matrimonio anterior ha de estimarse perjudicada (luego no es el sujeto pasivo del delito) y el contrayente de buena fe del segundo matrimonio (nuevamente calificada como persona afectada por el delito), concluyendo que el delito de bigamia es un delito pluriofensivo.

10 ESCRIHUELA CHUMILLA, F.J. Memento Penal La Ley Wolters Kluwer. 2016. Pág. 1061. 
liares derivadas del mismo ${ }^{11}$, con sus derechos y deberes, en tanto que pueden quedar afectadas por la situación matrimonial de los sujetos ${ }^{12}$.

Sin embargo, el segundo o ulterior cónyuge aparente no puede ser en ningún caso sujeto pasivo del delito de bigamia desde el momento en que, de estar en connivencia con el cónyuge bígamo, puede ser castigado como partícipe en este delito, como se explicará más adelante.

\subsection{Elementos del tipo}

\subsubsection{Conducta delictiva}

El precepto es claro, implica el contraer válidamente nupcias por segunda o en ulterior ocasión sin que haya sido disuelto ${ }^{13}$ un válido primigenio matrimonio, siendo conocedor de la citada situación el sujeto activo del tipo en calidad de autor.

El artículo 89 del CC establece que "la disolución del matrimonio por divorcio sólo podrá tener lugar por sentencia que así lo declare y producirá efectos desde su firmeza. No perjudicará a terceros de buena fe sino a partir de su inscripción en el Registro Civil".

Es decir, viene a ser preciso, en primer lugar la existencia de un primer matrimonio válido. Debe entenderse válido como aquél que ha sido celebrado en cualquiera de los ritos válidamente aceptados por el Derecho español (art. 49 CC). Así fundamentales, son los acuerdos del Estado español con la Iglesia Católica (Tratados sobre asuntos Jurídicos con la Santa Sede); con las Comunidades Israelitas de España (C.I.E.); con la Federación Islámica Española (F.C.I.); así como con la Federación de Entidades Religiosas Evangélicas de España (F.E.R.E.D.E.). A través de estos acuerdos se vienen a plasmar los automáticos efectos civiles de los matrimonios celebrados en cualesquiera de los ritos religiosos referenciados ${ }^{14}$.

De este modo, se viene a declarar judicialmente lo irrelevante que para el Derecho penal se refiere los matrimonios celebrados conforme a otros ritos religiosos o profanos, que no gocen del reconocimiento de los efectos civiles conforme a la Ley española. Así, no se han reconocido efectos al matrimonio celebrado por rito vudú, o bien un matrimonio celebrado en la Little White Wedding Chapel de la ciudad de Las Vegas, Estados Unidos, e inscrito en el Registro Civil del Condado de Clark, Nevada (SAP Málaga de 10 diciembre 2013 $)^{15}$.

El CP exige que se trate de un matrimonio legalmente previo, con independencia de la ulterior declaración de nulidad del mismo. De este modo, si celebrado el segundo matrimonio subsistiendo el primero, éste viene a declararse nulo con efectos ex tunc, no deberíamos plantear de un modo serio la ausencia de tipicidad de la conducta.

11 SAP Granada, Secc. 1a, 25 marzo 2003: el interés publico en asegurar el orden jurídico matrimonial establecido por el Estado; SAN (Sala de lo Penal), 18 diciembre 2013.

12 RODRÍGUEZ NÚÑEZ, A. "Parte especial de Derecho Penal”. Dykinson. 2016. Pág. 258.

13 Conforme determinan los arts. 85 y 73 a $80 \mathrm{CC}$, el matrimonio se disuelve legalmente por la muerte o por la declaración de fallecimiento de uno de los cónyuges y por sentencia firme de divorcio, o de nulidad, respectivamente.

14 SAP Melilla, Secc. 7, 11 febrero 2003; validez de un primer matrimonio islámico celebrado en Marruecos.

15 SAP Málaga, Secc. 1, 10 diciembre 2013 (Rec. 308/2013). 


\subsubsection{Dolo, Error de tipo y Error de prohibición}

De este modo, como indica el muy solvente trabajo de P. JuÁREZ PÉREZ ${ }^{16}$, varios datos previos deben tenerse presentes:

$\left.1^{\circ}\right)$ Este delito sólo puede cometerse en forma dolosa (= la expresión legal "a sabiendas" es elocuente de ello). Nadie discute la necesidad de la concurrencia del dolo directo o de primer grado, no siendo posible su comisión, quedando impune, por dolo eventual, la imprudencia o el error ${ }^{17}$.

Se exige la conciencia de la no disolución y subsistencia legal del anterior matrimonio. Nadie cree por error que está soltero si ha contraído matrimonio, y tampoco se puede ignorar que no se había obtenido el divorcio del anterior matrimonio, ya que nadie cree por error que está divorciado si no ha existido un proceso de divorcio (SAP La Coruña 28 septiembre 2001; SAP Málaga 11 febrero 2003; SAP Murcia 13 noviembre 2006; SAP Alicante 11 diciembre 2006). En las referidas resoluciones judiciales viene a apreciarse el dolo directo en los supuestos en los que el sujeto activo no distinguía entre la institución de la separación judicial del divorcio judicial. Los condenados entendían que a pesar de subsistir una sentencia de separación judicial podían contraer nuevas nupcias.

En el mismo sentido, el sujeto que conocía su anterior matrimonio en Alemania y conocía su separación ante el Juzgado de Villajoyosa. Igualmente conocía que no podía contraerse nuevo matrimonio sin haber obtenido el previo divorcio o ruptura del vínculo matrimonial precedente. Pese a su conocimiento, el acusado acudió al Registro Civil de Burgos para insta el inicio de un expediente de matrimonio y no solo no indicó que está separado sino que sus declaraciones a los efectos del art. 240 RRC fueron las siguientes: "Que su estado civil es soltero, lo cual no era cierto pues al menos constaba que era separado. Con tal falsedad manifiesta junto con la otra solicitante, -que no habían estado casados antes-, lo cual en el caso del recurrente no se ajustaba a la realidad, pues había estado casado con anterioridad" (SAP Burgos, 30 julio 2004).

De este modo, el dolo, en cualquiera de sus dimensiones, se excluye cuando el segundo o ulterior contrayente sufre un error de tipo, invocación muy generalizada en los procedimientos penales seguidos por el delito de bigamia, como señala E. RAMóN RIBAS $^{18}$.

Así, resoluciones, como se verá, en las que los acusados vienen a alegar el error de tipo, son la SAP Islas Baleares 18 diciembre 2000; SAP Murcia 13 noviembre 2006 y SAP Pontevedra 20 junio 2012.

$2^{\circ}$ ) Como ya me he referido, el error puede influir en la comisión del delito, pues es muy frecuente que el sujeto alegue que "no sabía" o "no era consciente" de que, en el momento de contraer un segundo matrimonio, el primer matrimonio que le afectaba era perfectamente válido en España (= error penal “de tipo").

A pesar de lo anterior, el acusado, mayor de edad, a sabiendas de que el matrimonio civil celebrado por él mismo con su primera esposa celebrado en Sidney, Nueva Gales

16 JUÁREZ PÉREZ, P. “Jurisdicción española y poligamia islámica: ¿Un matrimonio forzoso?”. REEI. Núm. 23. Junio 2021, pp.21-26.

17 CÓRDOBA RODA J. y GARCÍA ARÁN, M., "Comentarios al Código Penal. Parte Especial", Marcial Pons, Madrid, 2004, p. 548.

18 RAMÓN RIBAS, E. “Comentarios a la Parte Especial de Derecho Penal”. Aranzadi. 2016. Pp. 548-554. 
de Sur, Australia en fecha 17 septiembre 1984, no estaba legalmente disuelto por no haber obtenido sentencia firme de divorcio, en fecha 4 octubre 1997 contrajo matrimonio canónico en la Iglesia de Santa María La Mayor de Inca con una tercera persona. Todo ello a pesar de que en fecha 14 abril 1998, por el Juzgado de $1^{\text {a }}$ Instancia e Instrucción de Inca, fue dictada sentencia de divorcio respecto del primer matrimonio (SAP Islas Baleares 18 diciembre 2000). En el mismo sentido la SAP Murcia, 13 noviembre $2006^{19}$, donde se puso de manifiesto que el error que se alegó por el acusado carecía de acreditación, pues, como era conocido, ese error que excluye el dolo debe encontrarse seriamente fundado y demostrado por hechos evidentes que lo justifiquen. Confirmando lo anterior la SAP Pontevedra 20 junio 2012, determinado que cuando se alega un error invencible sobre un hecho constitutivo de infracción penal (tratándose de un matrimonio ilegal del art. 217 $\mathrm{CP}$ ) ha de ser totalmente acreditado. Viene a sustanciarse la desestimación recurso en atención al brocardo latino, la ignorancia de la Ley no excusa su cumplimiento, por lo que la apreciación del error debe provenir bien de las circunstancias personales de quien lo haya cometido -caso de personas de escasísima cultura- o bien de las posibilidades que se ha tenido de adquirir asesoramiento o instrucción, o de acudir a medios que le permitieran conocer la trascendencia jurídica de su obrar.

Por ello, como señala P. JuÁREz PÉREz, los tribunales penales españoles sólo pronuncian sentencias condenatorias por delito de bigamia cuando existe plena certeza de que el autor era plenamente consciente de la existencia de un matrimonio válido anterior y del carácter delictivo del segundo o ulterior matrimonio. Debe, por tanto, descartarse, en su caso, que concurra un "error de tipo".

En consecuencia, es frecuente que los acusados de delito de bigamia hagan valer circunstancias tales como que un matrimonio coránico celebrado en Marruecos no es válido en España (SAP Málaga 11 febrero 2003), o que el matrimonio anterior era nulo bien por haber sido calificado de "matrimonio de complacencia" (SAP Almería 29 enero 2009), o porque han transcurrido cinco años desde la separación conyugal (SAP Madrid 12 septiembre 2001);

$3^{\circ}$ ) El error de prohibición no es usual en este delito, pues es ampliamente conocido que, en el Derecho español, la bigamia constituye un delito castigado en el Código Penal (SAP León 22 octubre 2015 [delito de bigamia y Derecho dominicano]) ${ }^{20}$.

\section{EL LUGAR DE COMISIÓN DEL DELITO}

Este delito se considera cometido "en territorio español" a los efectos del art. 23.1 LOPJ y del art. $8 \mathrm{CC}$, cuando un sujeto está vinculado por un matrimonio que es válido en España, sea cual sea el país de su celebración, y no obstante, contrae otro matrimonio que es, en principio, aunque sólo aparentemente, pues como se ha dicho adolece de una nulidad radical, también válido en España.

19 SAP Murcia, Secc. 4, 13 noviembre 2006.

20 SAP León, Secc. 3, Rec. 943/2015: Se denuncia en el recurso indebida aplicación del artículo 217 del código penal por no concurrir el elemento subjetivo del tipo, realización de la conducta "a sabiendas", al actuar el apelante en la creencia de que cuando contrajo el segundo matrimonio en España se encontraba disuelto por divorcio el primer matrimonio que había contraído en su país de origen República Dominicana. 


\section{CONCLUSIÓN}

Por ello cabe indicar que:

$\left.1^{\circ}\right)$ Al margen de la falta de relevancia del hecho que los matrimonios hayan sido o no inscritos en el Registro Civil español, pues el matrimonio produce efectos legales desde el momento de su celebración (SAP Málaga, Melilla, Penal, 11 febrero 2003, SAP Coruña de 28 septiembre 2001); este tipo de delito, básicamente, solo viene a concurrir ante los matrimonios blancos o de conveniencia.

Se trata de un delito que podría considerarse obsoleto, en clara regresión, siendo totalmente prescindible en lo que a su tipicidad se refiere.

Bastaría con la aplicación del Código Civil, en atención a la simulación absoluta que supone, quedando latentes otros tipos penales, como los delitos de falsedad, o el abandono de familia.

$2^{\circ}$ ) Siendo el segundo matrimonio nulo de pleno derecho, pero el delito se habrá consumado porque este segundo enlace se contrajo por persona que, en dicho momento, estaba ligada por un anterior matrimonio válido en España (SAP Gerona 20 julio 2009, SAP Gerona 21 enero 2008);

$3^{\circ}$ ) Tampoco es relevante el país donde los matrimonios se hayan celebrado. Para que el delito de bigamia pueda entenderse como un delito "cometido en España", el segundo o posterior matrimonio debe afectar al sistema matrimonial monogámico "español", que es el bien jurídico protegido. Por ello, el delito de bigamia se produce "en España" cuando el matrimonio es aparentemente válido en España con arreglo a las normas españolas de Derecho internacional privado y uno de los contrayentes unidos por múltiple matrimonio ostenta "nacionalidad española" (AAP Cádiz 3 noviembre 2008 [nulidad de matrimonio contraído en Marruecos, aplicación de la Ley del país de celebración del matrimonio y posible delito de bigamia]), o el matrimonio se celebra "en territorio español". Si no concurre ninguna de las dos últimas circunstancias, debe entenderse que el segundo o ulterior matrimonio no afecta al sistema matrimonial español y la conducta de los sujetos ligados por múltiples matrimonios no se ha desarrollado "en España".

\section{BIBLIOGRAFÍA}

Álvarez García (Dir.)/Manjón-Cabeza Olmeda/Ventura Püschel (Coords.). PE I, 2a , 2011, 879; en: QUINTERO OLIVARES/CARBONELL MATEU/MORALES PRATS/GARCÍA RIVAS/ÁLVAREZ GARCÍA (dirs.)/MANJÓN-CABEZA OLMEDA/VENTURA PÜSCHEL (coords.), PE, 2011, 231.

Boix Reig/Jareño LeAL, en: Vives Antón (CoORd.).Comentarios CP I, 1996, 1047 s., analizando el concurso con la estafa y las falsedades.

Carbonell Mateu/González Cussac, en: Vives Antón/Orts Berenguer/Carbonell Mateu/GonzáLez Cussac/Martínez-Buján Pérez, PE, $3^{\mathrm{a}}, 2010,381$, analizando el concurso con la estafa; MORETÓN TOQUERO, Matrimonios ilegales, 2001, 17, planteando el concurso con abandono de familia (y con el de falsedades mencionando las dudas sobre el tratamiento concursal).

Conde-Pumpido Tourón (Dir.)/López Barja De Quiroga (Coord.), CP III, 2007, 1650, analizando el concurso con la falsedad documental (a favor del concurso de leyes para evitar la excesiva punibilidad de aplicarse las reglas del concurso de delitos); CARRASCO ANDRINO, en: LLP 66 (2009), $14 \mathrm{~s}$., analizando los concursos con las falsedades (de delitos si la falsedad es previa a la bigamia, de leyes si es posterior), abandono de familia y estafa. 
Córdoba Roda J. y García Arán, M., “Comentarios al Código Penal. Parte Especial”, Marcial Pons, Madrid, 2004, p. 548.

Cugat Mauri, en: Córdoba Roda/García Arán (Dirs.). Comentarios CP PE I, 2004, 544, 551; MUÑOZ SÁNCHEZ, en: DÍEZ RIPOLLÉS/ROMEO CASABONA (coords.), Comentarios CP II, 2004, 1076 ss.

Escrinuela Chumilla, F. J. Memento Penal La Ley Wolters Kluwer. 2016. Pág. 1061.

JuÁrez PÉREZ, P. “Jurisdicción española y poligamia islámica: ¿Un matrimonio forzoso?”. REEI. Núm. 23. Junio 2021, pp.21-26.

Labaca Zabala, M. L. "El delito de bigamia", Revista Electrónica Derecho Penal Online (disponible en http://www.derechopenalonline.com).

LAmarca Pérez (CoOrd.), PE, 6 ${ }^{\mathrm{a}}, 2011,229$, planteando el concurso con la estafa y las falsedades documentales (cuando la falsedad es medio para cometer la bigamia).

Manzanares Samaniego. CP II, 2010, 363, planteando el concurso con la estafa, las falsedades documentales y los delitos contra la libertad sexual; GÓMEZ MARTÍN, en: CORCOY BIDASOLO/ MIR PUIG (dirs.), Comentarios CP, 2011, 496, analizando el concurso con el delito de abandono de familia (concurso de leyes a favor del delito de bigamia por principio de consunción), en: CORCOY BIDASOLO (dir.)/VERA SÁNCHEZ (coord.), PE I, 2015, 449; GORDILLO ÁLVAREZ-VALDÉS.

PÉrez FERrer, F. “Análisis de los problemas interpretativos y aplicativos del delito de bigamia en el Derecho Penal español”, Revista Electrónica. Ciencia Penal y Criminología”, núm. 22, 2020. P.p. 11 y ss.

Ramón Ribas, E. “Comentarios a la Parte Especial de Derecho Penal”. Aranzadi. 2016. Pp. 548-554.

Ramón Ribas, En: Quintero Olivares (Dir.)/Morales Prats (Coord.), PE, 9a , 2011, 558 s., 560.

Terradillos Basoco, en: Cobo Del Rosal (Dir.). Comentarios CP VII, 2003, 681; en: TERRADILLOS BASOCO (coord.), PE I, 2011, 302, analizando el concurso con la estafa y la falsedad documental, en este caso analizando la tesis jurisprudencial sobre la calificación del concurso como de leyes cuando la falsedad documental es posterior a la comisión del delito de bigamia.

Trapelo Barreales, M. A. "Matrimonios ilegales y derecho penal. Bigamia, matrimonio inválido, matrimonio de conveniencia, matrimonio forzado y matrimonio precoz. Los delitos de matrimonios ilegales: Arts. 217 y 218 CP”. Tiranto Lo Blanch. Marzo 2016, pp. 4 y ss.

Rodríguez NúÑez, A. “Parte especial de Derecho Penal”. Dykinson. 2016. Pág. 258.

SuÁrez González, en: Rodríguez Mourullo (Dir.)/Jorge Barreiro (CoOrd.). Comentarios CP, 1997, 646, analizando el concurso con los delitos de falsedades documentales; CARBONELL MATEU, en: H-Vidal Guitarte I, 1999, 174, analizando el concurso con la estafa y las falsedades documentales. 